\title{
Hemodynamic Consequence of Different Pacing Modes after Aortic Valve Replacement
}

\author{
Arndt $\mathrm{H}$ Kiessling, MD \\ MSB Medical School Berlin, Berlin, Germany
}

\section{ABSTRACT}

Objectives: Ventricular pacemaker stimulation may cause deterioration of hemodynamics in patients with left-ventricular hypertrophy following aortic valve replacement. Since the diastolic function is often impaired, it remains unclear which heart rate best optimizes cardiac output. Low heart rates are suggested to treat impaired diastolic function chronically, but it is possible that cardiac output may be augmented by increasing the heart rate in patients with a fixed stroke volume (SV). The aim of this study is the identification of the best pacing mode and heart rate for the surrogate parameter SV and cardiac index(CI) in patients with left ventricular hypertrophy.

Methods: Various pacemaker stimulation modes and different heart rates, as well as their influence on hemodynamics, were tested following aortic valve replacement in 48 patients with severe left-ventricular hypertrophy (Intraventricular septum (IVS) $>1.5 \mathrm{~cm}$ ) and aortic stenosis. SV and cardiac output were recorded by pulse curve analysis. Four modes of stimulation (right ventricular pacemaker stimulation (DDDright), left ventricular pacemaker stimulation (DDDleft), biventricular pacemaker stimulation (DDDbi), atrial pacemaker stimulation (AAI)) were documented at five different rates (60, $80,100,120,140$ beats/min) and three different postoperative time points (intraoperatively, $3 \mathrm{~h}$ and $24 \mathrm{~h}$ postoperatively).

Results: The highest CI was found at linear rates between 60 to $140 \mathrm{bpm}$. AAI was the best mode of stimulation in the majority of cases (35\%), but in others, either left, right and/ or biventricular stimulation was found to be better (15\%). SV showed a u-shaped trend with a peak at 100 beats $/ \mathrm{min}$.

Conclusion: An increase in the heart rate does not lead to a notable drop in SV postoperatively in left-ventricular hypertrophy; hence a rise in cardiac output can be anticipated up to a rate of 100 beats/min. A standardized response in terms of an ideal pacemaker stimulation mode could not be identified.

\section{INTRODUCTION}

Benefits of cardiac resynchronization therapy in patients with severe left ventricular (LV) dysfunction (ejection fraction $<35 \%$ ) have been established by several randomized controlled trials [García-Bengochea 2012; Auricchio 2007;

Received March 8, 2017; accepted April 17, 2017.

Correspondence: Arndt H Kiessling, MD, MSB Medical School Berlin, Hochschule für Gesundheit und Medizin Siemens Villa Calandrellistraße 1-9 12247 Berlin, Germany (e-mail: cardiac.surgeon@dr-kiessling.com).
Cazeau 2001]. It has been demonstrated that the use of left ventricular pacing (DDDleft) or biventricular (DDDbi) pacing improves cardiac function by generating a more efficient ventricular contraction. Reasons for this could be that asynchronous contraction shortened the diastolic filling times at defined and given heart rates, especially after cardiac surgical procedures [Bakhtiary 2007]. Thus, cardiac function is impaired after cardiopulmonary bypass (CPB).

Delayed recovery of myocardial performance following restoration of myocardial flow, known as "stunning," is a well-documented phenomenon associated with $\mathrm{CPB}$ reperfusion [Bolli 1990]. The adverse contraction of the inter-ventricular septum toward the right ventricle, during systolic contraction is another possible occurrence after CPB [Reynolds 2007]. The aforementioned risks, and possible depressed preoperative ventricular function, are often responsible for hemodynamic instability. Weaning off of $\mathrm{CPB}$ requires inotropic or vasoactive mechanical support, or temporary pacemaker stimulation. The placement of right ventricular (RV) temporary epicardial electrode is routine in patient care during open cardiac surgery procedures to treat bradycardia or an atrioventricular block. However, the pacing site placement usually generates an undesirable cardiac effect.

Cannesson et al. [Cannesson 2009] have shown that acute right atrium-rapid ventricular pressure (RA- RVP) after CPB, in the absence of right bundle branch block (RBBB), worsens cardiac output. Several authors [Bakhtiary 2007; Nelson 2000] have demonstrated that DDDleft or DDDbi can acutely improve systolic function in cases with intraventricular conduction delay. Dzemali et al found that DDDbi was associated with improved left ventricular (LV) contractility without raising MeanV02 when compared with atrial pacing [Bakhtiary 2007]. This fact may allow for the diminishing of the inotropic support, additionally protecting myocardial metabolism. Therefore, optimization of temporary pacing after $\mathrm{CPB}$, particularly in cases with depressed $\mathrm{LV}$ function, is now a co-adjuvant method for the improvement of cardiac output. Despite this, patient subgroups could still be identified as non-responders to DDDbi or have greater difficulty finding the optimized pacing mode in postoperative care. However, there is no conclusive evidence to indicate what hemodynamic improvements should be the goal for patients with left ventricular hypertrophy.

Clinically, ventricular pacemaker stimulation is found to cause a clear deterioration in the hemodynamic of many patients with significant LV hypertrophy following aortic valve replacement. Since the diastolic function is 


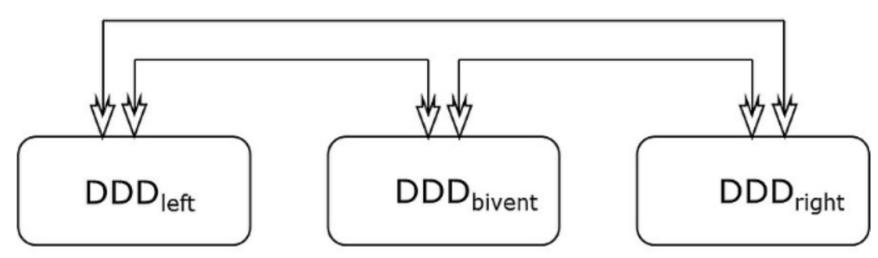

Figure 1. Different pacing modes.

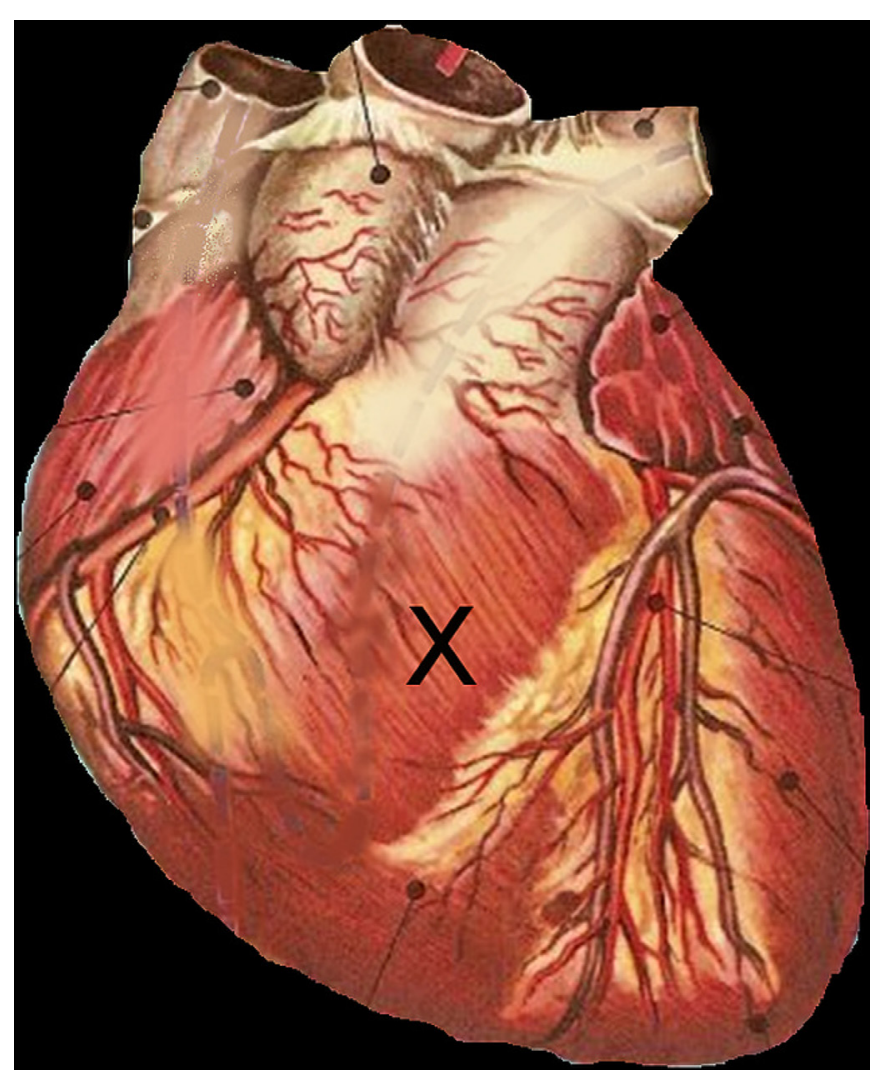

Figure 2. Pacemaker wire placement.

often impaired, the question also arises as to the heart rate which would be most suitable for optimizing the cardiac output. Although low heart rates tend to be suggested for treating impaired diastolic function, cardiac output can be augmented by increasing the heart rate perioperatively with a fixed stroke volume (SV). The pressure overload in patients with severe aortic stenosis results in concentric $L V$ hypertrophy. This has a negative impact on diastolic function and reduced relaxation rate.

The present study wants to prove the hypothesis that sequential biventricular stimulation substantially improves the hemodynamic status of patients with severe LV hypertrophy after aortic valve replacement. Even though lower heart rates are more commonly recommended when treating diastolic dysfunction, cardiac output can be increased over the perioperative period by raising the heart rate with a fixed SV. The aim of the study is the identification of the best pacing
Table 1. Inclusion and Exclusion Criteria

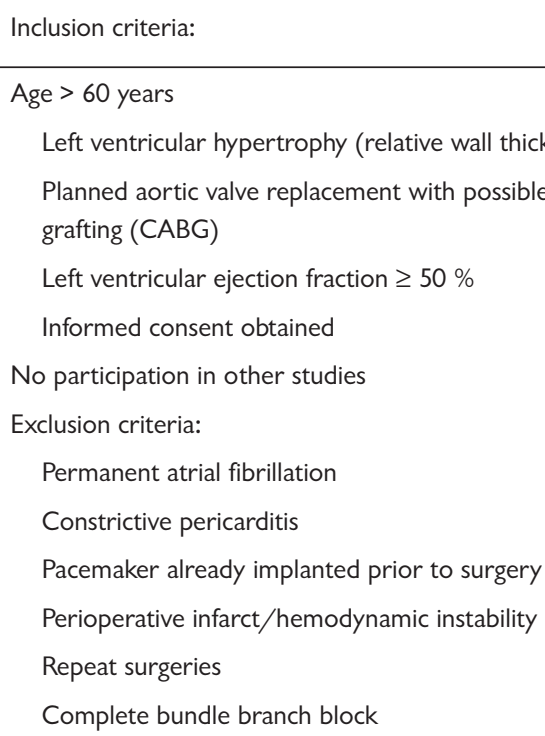

IVS indicates intraventricular septum.

mode and heart rate for the surrogate parameter SV and CI in patients with $L V$ hypertrophy.

\section{METHODS}

\section{Patient Selection}

Between January, 2011, and December, 2013, 48 patients were included in the open-label, unblinded, monocentric, non-randomized (consecutive) treatment study. The purpose of this study was to show intra-individual differences between different forms of pacemaker stimulation in patients with ventricular hypertrophy who underwent non-urgent aortic valve replacement. The primary endpoints were hemodynamic parameters (cardiac index (CI) and SV) recorded during the postoperative period. Data for four stimulation modes were collected and compared for each individual patient (intra-individual comparison): right ventricular pacemaker stimulation (DDDright), DDDleft, DDDbi, and atrial pacemaker stimulation (AAI) (Figure 1). Patients were selected by preoperative inclusion and exclusion criteria (Table 1).

\section{Study Design}

The study protocol was reviewed and given a positive vote by the institutional Ethics Committee of the Goethe University (no.19/10) (NCT01081093). Written informed consent was obtained from all patients. Blinding of the study was impossible. The statistical evaluation and endpoint evaluation was also done without blinding. A power analysis in this proof of concept study was not carried out, due to the fact that comparable data in this selected patient group were not available. Patients were screened one to seven days before open aortic valve replacement and gave informed consent at that time. 
Table 2. Preoperative demographic data and risk scores.

\begin{tabular}{lcc}
\hline & $\mathrm{n}$ & $\%$ \\
\hline Male & $10(48)$ & 69 \\
Diabetes & $11(48)$ & 23 \\
Dyslipidaemia & $18(48)$ & 38 \\
Hypertension & $33(48)$ & 70 \\
Permanent atrial fibrillation* & $0(48)$ & 0 \\
Current smoker & $1(48)$ & 2 \\
& Mean \pm Standard Deviation & Min-max \\
Age (y) & $73 \pm 5$ & $65-90$ \\
Weight (kg) & $78 \pm 15$ & $50-130$ \\
Height (cm) & $172 \pm 9$ & $148-193$ \\
BMl & $26 \pm 3.7$ & $17-38$ \\
Euroscore & $4.6 \pm 2.1$ & $0-11$ \\
Intraventricular septum (cm) & $1.9 \pm 0.3$ & $1.5-2.3$ \\
QRS duration (mm) & $0.9 \pm 0.1$ & $0.7-1.2$ \\
\hline
\end{tabular}

*Atrial fibrillation is an exclusion criteria.

Cardiac surgery was performed in accordance with accepted surgical practice.

\section{Pacing Protocol}

Before weaning from cardiopulmonary bypass, temporary epicardial pacing wires (unipolar pacing lead wire, Osypka AG, Rheinfelden-Herten, Germany) were sutured into the roof of the atrium close to the superior vena cava inflow, into the mid-right ventricle (3-4cm right of LAD), and into the mid-left ventricle (left circumflex distribution) (Figure 2). A DDD temporary external pulse generator (PACE 300 Osypka AG, Rheinfelden-Herten, Germany) was used. The PACE 300 is an external pacemaker for temporary cardiac resynchronization therapy (CRT) with one atrial and two independent ventricular channels. The resynchronization of both ventricles is achieved with selective trigger and output, and the adjustable intraventricular stimulation delay (fixed protocol AV delay: 120ms). Pacing thresholds were tested to confirm lead function and capture. In cases of diaphragmatic contractions, the lead was covered by a sponge (TachoSil Takeda, Berlin, Germany) to have a better electric insulation. Pacing with the assigned modalities was only commenced when stable conditions of vasopressors, inotropes, and volume therapy were achieved after the end of cardiopulmonary bypass, four to six hours after surgery, and 24 hours after surgery. The standardized pacing protocol for each patient was AAI, DDDright, DDDleft, DDDbi starting with 80 beats/minute. CI and SV were measured by pulse contour analysis (Vigileo/FloTrac, Edwards Lifescience, Irvine, USA). Then, the pacing series was repeated with 100 beats/minute. Only for the best pacing mode (highest CI) were frequencies of 60, 120 and 140 beats/minute tested as well.

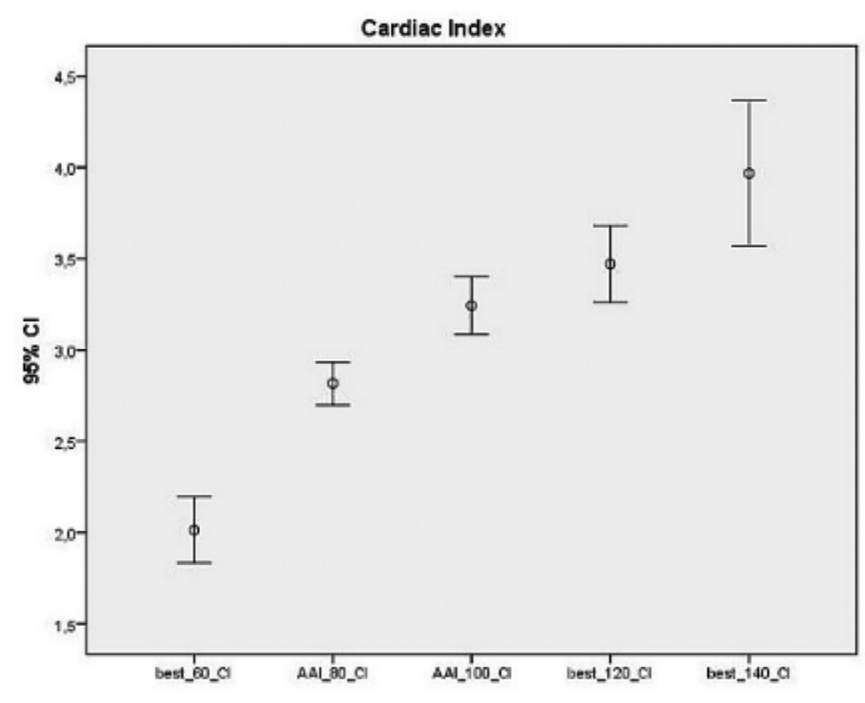

Figure 3. Direct correlation of heart rate and cardiac index.

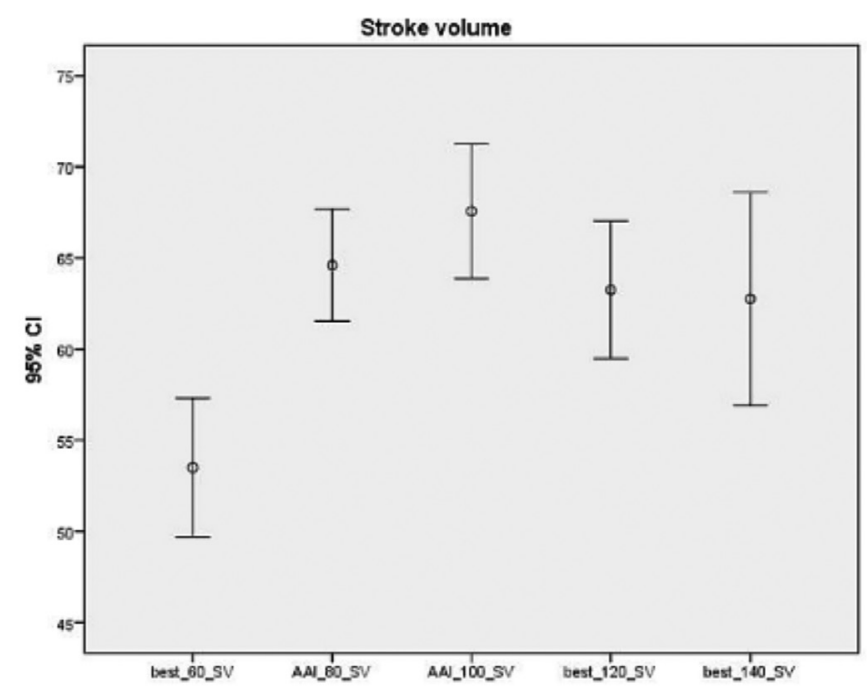

Figure 4. Parable curve of stroke volume. Increase of stroke volume from 60 beats $/ \mathrm{min}$ to 100 beats $/ \mathrm{min}$, and decrease in frequencies of 120 beats/min and 140 beats/min.

\section{Anaesthetic Protocol}

After routine monitoring was initiated, general anaesthesia was induced: $0.3-1 \mu \mathrm{g} / \mathrm{kg}$ sufentanil (Sufenta ${ }^{\circledR}$, JanssenCilag GmbH, Neuss, Germany), $1-2.5 \mathrm{mg} / \mathrm{kg}$ propofol (Disoprivan ${ }^{\circledR}$, AstraZeneca GmbH, Wedel, Germany) and $0.6 \mathrm{mg} / \mathrm{kg}$ rocuronium (Esmeron ${ }^{\circledR}$, Essex $\mathrm{GmbH}$, Munich, Germany). For maintenance of general anaesthesia, all patients received 1-2 vol\% sevoflurane (Sevoran ${ }^{\circledR}$, Abbott, Wiesbaden, Germany) and intermittent boluses of sufentanil. Isotonic crystalloid (Sterofundin ${ }^{\circledR}$, B. Braun GmbH, Melsungen, Germany) was infused perioperatively based on institutional standards. A Flotrac ${ }^{\mathrm{TM}}$ (Edwards Lifescience, Irvine, USA) sensor kit was connected to the radial arterial line and coupled with the Vigileo ${ }^{\mathrm{TM}}$ (Edwards Lifescience, 
Table 3. Stimulation Results

\begin{tabular}{|c|c|c|c|c|c|c|}
\hline & & 80 & 100 & 60 & 120 & 140 \\
\hline \multirow[t]{2}{*}{ AAl } & $\mathrm{Cl}(\mathrm{l} / \mathrm{min})$ & $2.8 \pm 0.56$ & $3.2 \pm 0.73$ & $2.0 \pm 0.56$ & $3.4 \pm 0.63$ & $3.9 \pm 1.46$ \\
\hline & $\mathrm{SV}(\mathrm{ml})$ & $65 \pm 15$ & $68 \pm 17$ & $53 \pm 11.6$ & $63 \pm 18$ & $63 \pm 20$ \\
\hline \multirow[t]{2}{*}{$\mathrm{DDD}_{\text {left }}$} & $\mathrm{Cl}(\mathrm{l} / \mathrm{min})$ & $2.7 \pm 0.52$ & $3.2 \pm 0.73$ & & & \\
\hline & $\mathrm{SV}(\mathrm{ml})$ & $63 \pm 14$ & $66 \pm 15$ & & & \\
\hline \multirow[t]{2}{*}{$\mathrm{DDD}_{\mathrm{bi}}$} & $\mathrm{Cl}(\mathrm{l} / \mathrm{min})$ & $2.8 \pm 0.48$ & $3.3 \pm 0.85$ & & & \\
\hline & $\mathrm{SV}(\mathrm{ml})$ & $65 \pm 14$ & $69 \pm 17$ & & & \\
\hline
\end{tabular}

$\mathrm{Cl}$ refers to cardiac index, SV, stroke volume. In 35\% of cases, AAI was the form of stimulation with the best measurement values (highest CI). DDDright was the best stimulation mode for $15 \%$ of patients, as were DDDleft and DDDbi.

Irvine, USA) monitor with an updated software version. Individual patient data (age, gender, body weight, height) were entered. After checking the arterial line waveform fidelity, the system was zeroed at mid-axillary level to calibrate pressure.

\section{Perfusion Technique}

The extracorporeal circuit included a membrane oxygenator (Quadrox ${ }^{\circledR}$ oxygenator, Maquet Cardiopulmonary AG, Hirrlingen, Germany) and a roller pump system (HL20 Maquet Cardiopulmonary AG) equipped with a cardioplegia heat exchanger (Plegiox®, Maquet Cardiopulmonary AG). During $\mathrm{CPB}$, non-pulsatile flow was maintained at 2.6-3 L/ $\mathrm{min} / \mathrm{m}^{2}$ and mean arterial blood pressure was maintained at $50-70 \mathrm{mmHg}$ by administration of norepinephrine (Arterenol $^{\circledR}$, Sanofi-Aventis GmbH, Hoechst, Germany). Extracorporeal circulation was performed under mild hypothermia $\left(32^{\circ} \mathrm{C}\right)$. On completion of surgery, the patients were rewarmed and weaned from CPB.

\section{Statistics}

Pre- and perioperative data were summarised as mean and standard deviation (SD) or median and 25 th- 75 th percentile if continuous, or as counts and percent if categorical. Continuous variables were tested for normality with the Kolmogorov-Smirnov test and compared between the four treatment groups (DDDright, DDDleft, DDDbi, AAI) with the Student's t-test or the Mann-Whitney U test, accordingly. Categorical variables were compared with the Fisher's exact test. Ordinal variables were also compared with the Mann-Whitney U test. Repeated measures analysis of variance (ANOVA) was used to compare serial data. Comparisons were considered significant if $P<.05$. Statistical analysis was performed by the Statistical Package for Social Sciences (SPSS) programme for Windows, version 21.0 (SPSS, IBM, USA).

\section{RESULTS}

\section{Patient Demographics}

The clinical characteristics of the patient population are summarized in Table 2. The majority of patients enrolled were male with a mean age of $73 \pm 5$ years $(\min 65 y$ - max $90 \mathrm{y})$ due to the fact that the inclusion criteria selected patients above the age of 65 . In all cases, a biological valve (Perimount, Edwards Lifescience, Irvine, USA) was implanted. In three cases additional bypass grafting was performed. All patients underwent surgery on cardiopulmonary bypass. The mean $\mathrm{LV}$ ejection fraction was $58 \% \pm 4 \%$. The preoperative electrocardiograms were without any significant bundle branch blocks or significant intraventricular conduction delay.

\section{Hemodynamic Testing}

All patients completed intraoperative testing and the early postoperative test, but only 28 of the 48 patients (58\%) completed hemodynamic testing at all three time points and pacing modalities. Testing was not completed because of complete heart block in four patients, lead capture failures in two patients, postoperative atrial fibrillation in four patients, painful pacing stimulation in five patients, removal of the arterial radial line for Vigileo measurements $(n=8)$, hemodynamic instability $(\mathrm{n}=1)$ or high spontaneous heart rate $(>80$ beats/min) in eight patients. For this reason we summarized the results and calculated mean values for all three postoperative time points of stimulation.

The results of the mean testing are shown in Table 3. Starting with a heart rate of 80 beats/min, all pacing modes (AAI, DDDright, DDDleft, DDDbi) were approximately identical for CI (min: $2.71 / \mathrm{min}$, max: 2.81/min), and there was no significant difference in SV between AAI and DDDbi pacing. The PACE 300 was adjusted to 100 beats/min, and the hemodynamic measurements were identical to the frequencies of 80. There were marginally better test results for DDDbi and AAI in SV and CI without reaching any significant level. No 
clear evidence was found as to whether between 80 beats $/ \mathrm{min}$ or 100 beats/min was the better pacing mode in heart beats. Only in $35 \%$ of cases was AAI the form of stimulation with the highest CI. DDDbi was the best stimulation mode for $15 \%$ of patients, as were DDDleft and DDDright. In these 17 subjects, we paced with additional 60 beats/min, 120 beats/ min and 140 beats/min.

For the AAI mode, we can see a direct correlation between heart rate and CI. The rational for this result is founded in its formula. $\left(\mathrm{CI}=\left(\mathrm{SV}^{*}\right.\right.$ heart rate)/Body surface area). The $\mathrm{CI}$ was doubled by increasing the pacemaker rate from 60 beats $/ \mathrm{min}$ to 140 beats/min (Figure 3). However, SV showed a different rate depenceny. Starting at 60 beats $/ \mathrm{min}$ with $53 \pm 11 \mathrm{~mL}$, the $S V$ continuously increased till a heart rate of 100 beats/min $(68 \pm 17 \mathrm{~mL})$ but decreased at higher rates (120 beats/min and 140 beats $/ \mathrm{min})$ to $63 \pm 20 \mathrm{~mL}(P$ $<.05)$ (Figure 4). Furthermore, most of the patients did not accept tachycardia of 140 beats/min longer than one minute by a drop of systolic blood pressure of more than $30 \%$, compared to pacing at 80 beats/min.

\section{DISCUSSION}

Temporary stimulation with RV epicardial electrodes after cardio-pulmonary bypass is a common practice after cardiac surgical procedures. In addition, the use of biventricular pacing in patients with reduced $\mathrm{LV}$ function or left bundle block to optimize the cardiac output is performed in several cardiac centers. This study focused on different patients in the same setting: patients with ventricular hypertrophy caused by severe aortic stenosis, and planned elective aortic valve replacement. We compared the postoperative changes of cardiac output and SV during right, left, biventricular, and atrial stimulation. Our results will be discussed in three main topics: first, the finding that no pacing mode was found to be optimal for all patients, with a high number of nonresponders to DDDbi, second, the possible explanation for the reduced $\mathrm{SV}$ in higher frequencies, and third, the question of whether there is an impact of the sites of temporary stimulation to optimize the cardiac output.

The strategy for perioperative and postoperative optimization by implanting an additional temporary pacing lead to initiate a DDDbi pacing mode is still controversial. Vaughan et al concluded in his review that a significant increase of the CI could be found in up to $22 \%$ of the patients with DDDbi pacing mode [Vaughan 2009]. Important for the interpretation of our results is the separation of the results of patients with CRT implantation, and patients after cardiac procedures and epicardial temporary placing. These patient groups seem not to be comparable. Furthermore, it is crucial to note the underlying cardiac disease. No significant hemodynamic improvement was found in subjects with normal ventricular function [Evonich 2008; Schmidt 2007], and the greatest benefits were observed in patients with low ejection fraction and a wide QRS complex [Vaughan 2009; Bakhtiary 2007; Weisse 2002].

In our trial, the patients had normal $\mathrm{LV}$ function with an ejection fraction of $>50 \%$ (inclusion criteria). This seems to be one reason why DDDbi pacing was the best pacing mode in only $15 \%$ of our test series. Our results failed to demonstrate consistent superiority of DDDbi when applied to the postoperative patient care. Evonich et al focused on patients with reduced $\mathrm{LV}$ function, but found results nearly identical to those of our series of patients with hypertrophic myopathy [Evonich 2008]. DDDbi resulted in various hemodynamic responses among individuals, with $17 \%$ of patients demonstrating improved hemodynamics, and $41 \%$ exhibiting worsened measurements. Moreover, both Evonich et al and our trials were unable to identify clinical outcome variables, because the studies were not adequately powered for this question [Evonich 2008].

The observations from the present proof of concept trial are not consistent with the several small non-randomized surgical studies that suggest a hemodynamic benefit in patients undergoing cardiac surgery [Weisse 2002; Saxon 1998; Foster 1995]. The difference of findings among studies are likely due to dissimilar study populations, pacing protocols, and methodologies for hemodynamics, clinical data acquisition, and optimal placement of the pacing electrodes [Rossillo 2004]. The pacing protocols of Evonich's study required epicardial leads to be positioned in the mid right and mid left ventricle along the distribution of the circumflex coronary artery [Evonich 2008]. In contrast, previous studies used paraseptal, posterobasal, apical, diaphragmal or outflow tract LV and RV lead placement [Weisse 2002; Saxon 1998]. A true synchronous pacing with respect to possible myocardial scar areas in CABG cases could not be achieved in all these scenarios.

The improvement of LV systolic synchronicity is a major component of cardiac resynchronization therapies. Yu et al found in an echocardiography and tissue Doppler imaging trial an improvement of systolic parameters only during DDDbi pacing [Yu 2003]. The LV volume was decreased acutely during DDDbi pacing, and a depressed diastolic function was observed in DDDright, DDDleft, and DDDbi pacing. The immediate volume unloading effect could be related to hemodynamic benefits, the reduction of mitral regurgitation, and the improvement of systolic synchronicity with higher efficiency of systolic emptying and reduced diastolic stress of the ventricle. Wachter et al shows that frequent-dependent upregulation of cardiac output is blunted in heart failure patients with preserved ejection fraction [Wachter 2009]. He reports that a progressive decrease of end-diastolic volumes at higher heart rates results in the reduction of SV and the blunting of the frequency-dependent up-regulation of cardiac output. Previous studies analysing $\mathrm{LV}$ volumes at increasing heart rates showed conflicting results in healthy subjects, with either decreased or no change in systolic volumes during pacing [Lui 1993; Erbel 1984; Dehmer 1983; Hung 1981.

The role of the heart rate in our patients is contradictory to common assumption. At least during the early postoperative course, a gain in cardiac output and SV could be observed up to a rate of 100 beats/min. For chronic diastolic heart failure, lowering of heart rate was found to improve symptoms and survival. Higher rates have been found to be unable to increase output in heart failure patients with preserved ejection fraction [Wachter 2009]. However, this finding is inconsistent with our results, which show a continuous increase of $\mathrm{CI}$ and SV, with a maximum at 100 beats $/ \mathrm{min}$ and a decrease 
in higher frequencies in patients with ventricular hypertrophy. It is still unclear in our selected cohort as to what degree the diastolic dysfunction has an impact on the outcome variables $\mathrm{CI}$ and SV. However, in the echocardiographic sub-study of the CHARM trail in normal ejection fraction heart failure patients, it was found that $33 \%$ of the group had no evidence of diastolic dysfunction, and moderate/severe diastolic dysfunction was found in only $44 \%$ of the patients [Persson 2007]. The definition of incomplete relaxation in humans is not well standardized, and the need for a consensus paper is obvious. Only with both of these conditions met would clinical trials on different cardiac pathologies be comparable.

\section{Limitations and Outlook}

There were several limitations and deficiencies in our trial. First, the present study was a pilot study by design, and therefore we did not have a large enough population to achieve adequate statistical power to detect small differences. In addition, the high drop out rate at different time points makes it difficult to draw clear conclusions about the best pacing mode. Nonetheless, our work represents the largest trial in this selected sub-group of patients with left ventricular hypertrophy. Missing data about the dys-synchrony in echocardiographic data is an additional limitation, and the selected time points of measurements were in a hemodynamic unstable, dynamic period. The intra-operative testing was restricted by instability of the patients with frequencies $>120$ beats $/ \mathrm{min}$.

\section{Acknowledgments}

The authors would like to express their particular thanks to the Study Coordinator Mrs.Sonja Friese for data acquisition and to the perfusionists of Maquet Services.

\section{REFERENCES}

Auricchio A, Metra M, Gasparini M, et al. 2007. Multicenter Longitudinal Observational Study (MILOS) Group: long-term survival of patients with heart failure and ventricular conduction delay treated with cardiac resynchronization therapy. Am J Cardiol 99:232-8.

Bakhtiary F, Dogan S, Dzemali O, et al. 2007. Impact of different pacing modes on left ventricular contractility following cardiopulmonary bypass. Pacing Clin Electrophysiol 30:1083-90.

Bolli R. 1990. Mechanism of myocardial "stunning". Circulation. 82:723-38.

Cannesson M, Farhat F, Scarlata M, Cassar E, Lehot JJ. 2009. The impact of atrio-biventricular pacing on hemodynamics and left ventricular dyssynchrony compared with atrio-right ventricular pacing alone in the postoperative period after cardiac surgery. J Cardiothorac Vasc Anesth 23:306-11.

Cazeau S, Leclercq C, Lavergne T, et al. 2001. Multisite Stimulation in Cardiomyopathies (MUSTIC) Study Investigators: effects of multisite biventricular pacing in patients with heart failure and intraventricular conduction delay. N Engl J Med 344:873-80.

Dehmer GJ, Firth BG, Nicod P, Lewis SE, Hillis LD. 1983. Alterations in left ventricular volumes and ejection fraction during atrial pacing in patients with coronary artery disease: assessment with radionuclide ventriculography. Am Heart J 106:114-24.
Erbel R, Schweizer P, Krebs W, Langen HJ, Meyer J, Effert S. 1984. Effects of heart rate changes on left ventricular volume and ejection fraction: a 2-dimensional echocardiographic study. Am J Cardiol 53:590-7.

Evonich RF, Stephens JC, Merhi W, et al. 2008. The role of temporary biventricular pacing in the cardiac surgical patient with severely reduced left ventricular systolic function. J Thorac Cardiovasc Surg 136:915-21.

Foster AH, Gold MR, McLaughlin JS. 1995. Acute hemodynamic effects of atrio-biventricular pacing in humans. Ann Thorac Surg. 59:294-300.

García-Bengochea JB, Fernández AL, Calvelo DS, Escudero JA, Gude F, Juanatey JR. 2012. Temporary epicardial left ventricular and biventricular pacing improves cardiac output after cardiopulmonary bypass. J Cardiothorac Surg Oct 22:113.

Hung J, Kelly DT, Hutton BF, Uther JB, Baird DK. 1981. Influence of heart rate and atrial transport on left ventricular volume and function: relation to hemodynamic changes produced by supraventricular arrhythmia. Am J Cardiol 48:632-8.

Liu CP, Ting CT, Lawrence W, Maughan WL, Chang MS, Kass DA. 1993. Diminished contractile response to increased heart rate in intact human left ventricular hypertrophy. Systolic versus diastolic determinants. Circulation 88:1893-906.

Nelson GS, Berger RD, Fetics BJ, et al. 2000. Left ventricular or biventricular pacing improves cardiac function at diminished energy cost in patients with dilated cardiomyopathy and left bundle-branch block. Circulation 102:3053-9.

Persson H, Lonn E, Edner M, et al. 2007. Investigators of the CHARM Echocardiographic Substudy-CHARMES. Diastolic dysfunction in heart failure with preserved systolic function: need for objective evidence: results from the CHARM Echocardiographic Substudy-CHARMES. J Am Coll Cardiol 49:687-94.

Reynolds HR, Tunick PA, Grossi EA, Dilmanian H, Colvin SB, Kronzon I. 2007. Paradoxical septal motion after cardiac surgery: a review of 3,292 cases. Clin Cardiol 30:621-3.

Rossillo A, Verma A, Saad EB, et al. 2004. Impact of coronary sinus lead position on biventricular pacing: mortality and echocardiographic evaluation during long-term follow-up. J Cardiovasc Electrophysiol 15:1120-5.

Saxon LA, Kerwin WF, Cahalan MK, et al. 1998. Acute effects of intraoperative multisite ventricular pacing on left ventricular function and activation/contraction sequence in patients with depressed ventricular function. J Cardiovasc Electrophysiol 9:13-21.

Schmidt C, Frielingsdorf J, Debrunner M, et al. 2007. Acute biventricular pacing after cardiac surgery has no influence on regional and global left ventricular systolic function. Europace 9:432-6.

Vaughan P, Bhatti F, Hunter S, Dunning J. 2009. Does biventricular pacing provide a superior cardiac output compared to univentricular pacing wires after cardiac surgery? Interact Cardiovasc Thorac Surg 8:673-8.

Wachter R, Schmidt-Schweda S, Westermann D, et al. 2009. Blunted frequency-dependent upregulation of cardiac output is related to impaired relaxation in diastolic heart failure. Eur Heart J 30:3027-36.

Weisse U, Isgro F, Werling Ch, Lehmann A, Saggau W. 2002. Impact of atrio-biventricular pacing to poor left-ventricular function after CABG. Thorac Cardiovasc Surg 50:131-5.

Yu CM, Lin H, Fung WH, Zhang Q, Kong SL, Sanderson JE. 2003. Comparison of acute changes in left ventricular volume, systolic and diastolic functions, and intraventricular synchronicity after biventricular and right ventricular pacing for heart failure. Am Heart J 145:E18. 